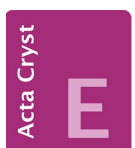

CRYSTALLOGRAPHIC COMMUNICATIONS

ISSN 2056-9890

Received 4 May 2015

Accepted 11 May 2015

Edited by M. Weil, Vienna University of Technology, Austria

Keywords: crystal structure; isotypism; chromium; caesium; diarsenate; channel structure

CCDC reference: 1400446

Supporting information: this article has supporting information at journals.iucr.org/e

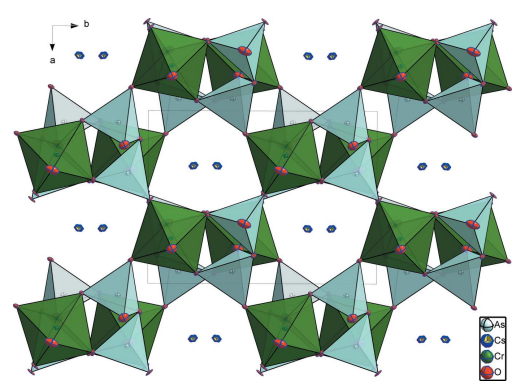

OPEN $\odot$ ACCESS

\section{Crystal structure of $\mathrm{CsCrAs}_{2} \mathrm{O}_{7}$, a new member of the diarsenate family}

\author{
Mohamad Alem Bouhassine and Habib Boughzala*
}

Laboratoire de Materiaux et Cristallochimie, Faculté des Sciences de Tunis, Université de Tunis El Manar, 2092 Manar II Tunis, Tunisia. *Correspondence e-mail: habib.boughzala@ipein.rnu.tn

Caesium chromium(III) diarsenate(V), $\mathrm{CsCrAs}_{2} \mathrm{O}_{7}$, was prepared by solid-state reactions. The title structure consists of isolated $\mathrm{CrO}_{6}$ octahedra and $\mathrm{As}_{2} \mathrm{O}_{7}$ diarsenate groups, sharing corners to build up a three-dimensional $\left[\mathrm{CrAs}_{2} \mathrm{O}_{7}\right]^{-}$ anionic framework. In this framework, channels extending parallel to [001] are present in which the ten-coordinate $\mathrm{Cs}^{+}$ions reside. $\mathrm{CsCrAs}_{2} \mathrm{O}_{7}$ is isotypic with the monoclinic $A^{\mathrm{I}} M^{\mathrm{III}} X_{2} \mathrm{O}_{7}\left(A^{\mathrm{I}}=\right.$ alkali metal; $\left.M^{\mathrm{III}}=\mathrm{Al}, \mathrm{Cr}, \mathrm{Fe} ; X=\mathrm{As}, \mathrm{P}\right)$ type I family of compounds crystallizing in the space group $P 2_{1} / c$.

\section{Chemical context}

In recent years, inorganic metal phosphates and arsenates with formula $A^{\mathrm{I}} M^{\mathrm{III}} X_{2} \mathrm{O}_{7}\left(A^{\mathrm{I}}=\right.$ alkali metal; $M^{\mathrm{III}}=\mathrm{Al}, \mathrm{Cr}, \mathrm{Fe} ; X=$ As, P) have been part of intensive research activities, with crystals grown either from high-temperature solid-state reactions or under aqueous solution conditions. The crystal chemistry of these compounds with $X_{2} \mathrm{O}_{7}$ groups reveals a large structural variety accompanied in some cases by interesting magnetic, electric, optical, or thermal expansion properties. Focusing on compounds with $M^{\mathrm{III}}=\mathrm{Cr}$, it is noticeable that corresponding diphosphates have been studied extensively, in contrast to the scarcely studied chromium diarsenates. Herein the preparation and crystal structure of

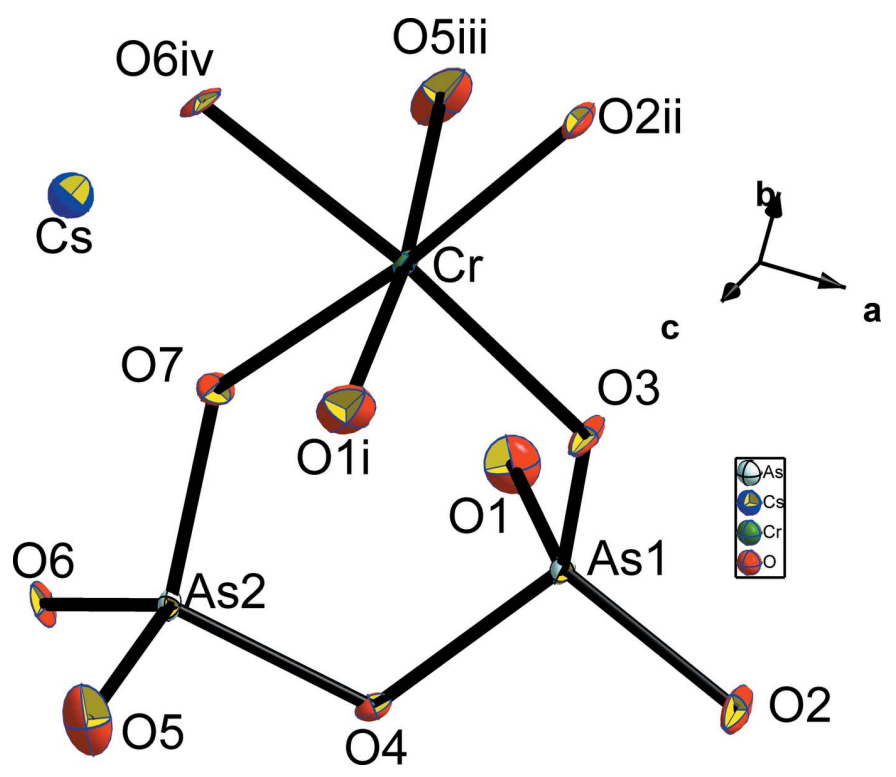

Figure 1

The coordination polyhedra around $\mathrm{Cr}$ and $\mathrm{As}$ atoms in the title structure. Displacement ellipsoids are drawn at the $50 \%$ probability level. [Symmetry codes: (i) $x, \frac{1}{2}-y, \frac{1}{2}+z$; (ii) $2-x, \frac{1}{2}+y, \frac{3}{2}-z$; (iii) $x, \frac{1}{2}-y$, $-\frac{1}{2}+z$; (iv) $1-x, \frac{1}{2}+y, \frac{3}{2}-z$.] 


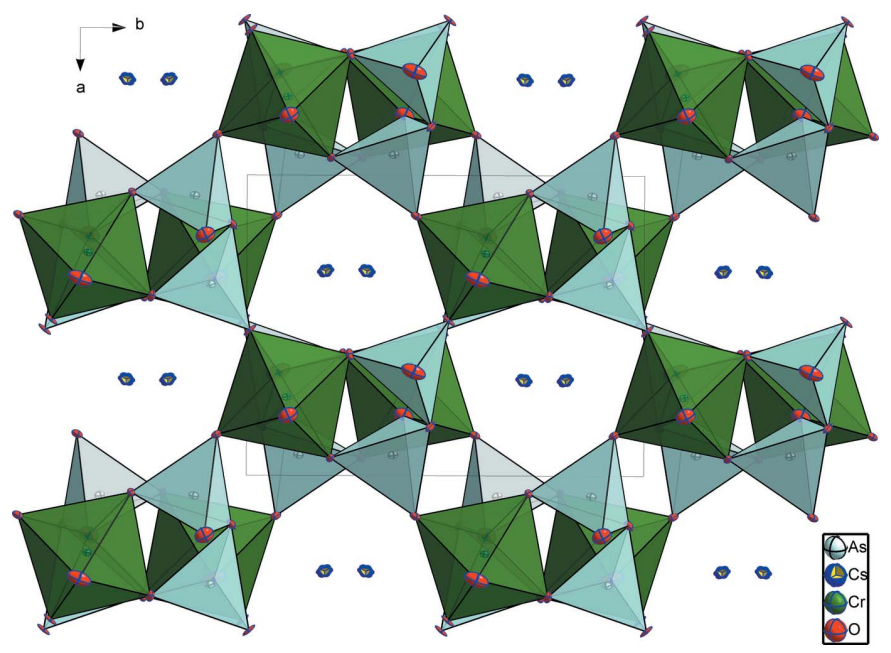

Figure 2

Projection of the $\mathrm{CsCrAs}_{2} \mathrm{O}_{7}$ structure showing the channels parallel to [001] in which the $\mathrm{Cs}^{+}$cations are located.

$\mathrm{CsCrAs}_{2} \mathrm{O}_{7}$ is reported, one of a series of new cesium chromium(III) arsenate compounds recently isolated by our group.

\section{Structural commentary}

The structure of $\mathrm{CsCrAs}_{2} \mathrm{O}_{7}$ can be described as a threedimensional $\left[\mathrm{CrAs}_{2} \mathrm{O}_{7}\right]^{-}$anionic framework (Fig. 1) with channels extending parallel to [001] that are occupied by tencoordinate $\mathrm{Cs}^{+}$cations (Fig. 2).

The two independent arsenic atoms form $\mathrm{AsO}_{4}$ tetrahedra and are connected via the bridging $\mathrm{O} 4$ atom into a diarsenate $\mathrm{As}_{2} \mathrm{O}_{7}$ anion. Like in the related structures of $\mathrm{KAlAs}_{2} \mathrm{O}_{7}$

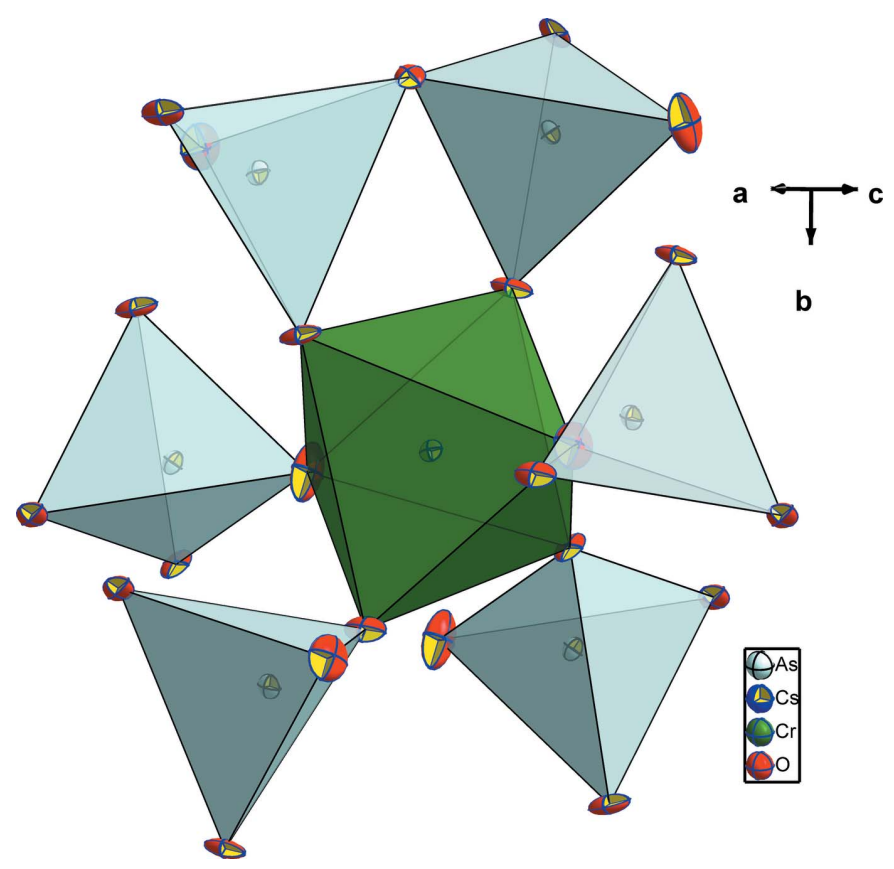

Figure 3

The environment of the $\mathrm{CrO}_{6}$ octahedron in the structure of $\mathrm{CsCrAs}_{2} \mathrm{O}_{7}$.
Table 1

Selected bond lengths $(\AA)$.

\begin{tabular}{llll}
\hline $\mathrm{Cr}-\mathrm{O}^{\mathrm{i}}$ & $1.944(4)$ & As1-O2 & $1.664(4)$ \\
$\mathrm{Cr}-\mathrm{O} 7$ & $1.954(4)$ & As1-O3 & $1.681(4)$ \\
$\mathrm{Cr}-\mathrm{O} 1^{\mathrm{ii}}$ & $1.978(4)$ & As1-O4 & $1.763(4)$ \\
$\mathrm{Cr}-\mathrm{O} 3$ & $1.982(4)$ & As2-O5 & $1.641(4)$ \\
$\mathrm{Cr}-\mathrm{O} 2^{\mathrm{iii}}$ & $2.007(4)$ & As2-O6 & $1.661(4)$ \\
$\mathrm{Cr}-\mathrm{O} 6^{\text {iv }}$ & $2.010(4)$ & As2-O7 & $1.669(4)$ \\
$\mathrm{As} 1-\mathrm{O} 1$ & $1.651(4)$ & As2-O4 & $1.750(4)$ \\
\hline
\end{tabular}

Symmetry codes: (i) $x,-y+\frac{1}{2}, z-\frac{1}{2}$; (ii) $x,-y+\frac{1}{2}, z+\frac{1}{2}$; (iii) $-x+2, y+\frac{1}{2},-z+\frac{3}{2}$; (iv) $-x+1, y+\frac{1}{2},-z+\frac{3}{2}$.

(Boughzala \& Jouini, 1995) and $\mathrm{RbAlAs}_{2} \mathrm{O}_{7}$ (Boughzala et al., 1993), the As-O distances involving the bridging $\mathrm{O} 4$ atom are the longest (Table 1). The As1-O4-As2 bridging angle of $118.7(2)^{\circ}$ in the title structure is somewhat smaller than that of $125.9(2)^{\circ}$ reported for the isotypic structure of $\mathrm{CsCrP}_{2} \mathrm{O}_{7}$ (Linde \& Gorbunova, 1982). The $\mathrm{O}-\mathrm{As}-\mathrm{O}$ bond angles span a range between $103.8(2)$ and $116.2(2)^{\circ}$ and $105.5(2)$ and $115.6(2)^{\circ}$, respectively, for As1 and As2, reflecting the distortion of each of the $\mathrm{AsO}_{4}$ tetrahedra. The $\mathrm{Cr}^{\mathrm{III}}$ cations are in a slightly distorted octahedral oxygen coordination with $\mathrm{Cr}-\mathrm{O}$ distances ranging from 1.944 (4) to 2.010 (4) $\AA$ (Table 1), and with $\mathrm{O}-\mathrm{Cr}-\mathrm{O}$ angles ranging from 82.96 (18) to $95.94(17)^{\circ}$ and from $172.37(19)$ to $173.72(17)^{\circ}$. Each $\mathrm{CrO}_{6}$ octahedron shares its corners with five $\mathrm{As}_{2} \mathrm{O}_{7}$ anions, one of which is chelating and the others belonging to four different $\mathrm{As}_{2} \mathrm{O}_{7}$ groups (Fig. 3). On the other hand, each $\mathrm{As}_{2} \mathrm{O}_{7}$ anion is surrounded by five $\mathrm{CrO}_{6}$ octahedra as depicted in Fig. 4. The environment of the ten-coordinate $\mathrm{Cs}^{+}$cation situated in the cavities of the resulting $\left[\mathrm{CrAs}_{2} \mathrm{O}_{7}\right]^{-}$framework is shown in Fig. 5.

It is worth mentioning that in the related aluminium diarsenate family $A^{\mathrm{I}} \mathrm{AlAs}_{2} \mathrm{O}_{7}\left(A^{\mathrm{I}}=\mathrm{K}, \mathrm{Rb}, \mathrm{Tl}, \mathrm{Cs}\right)$ (Boughzala \& Jouini, 1992) that crystallizes isotypically in space group $P \overline{1}$ and is classified as type II, the diarsenate groups have a different conformational orientation as those of the title

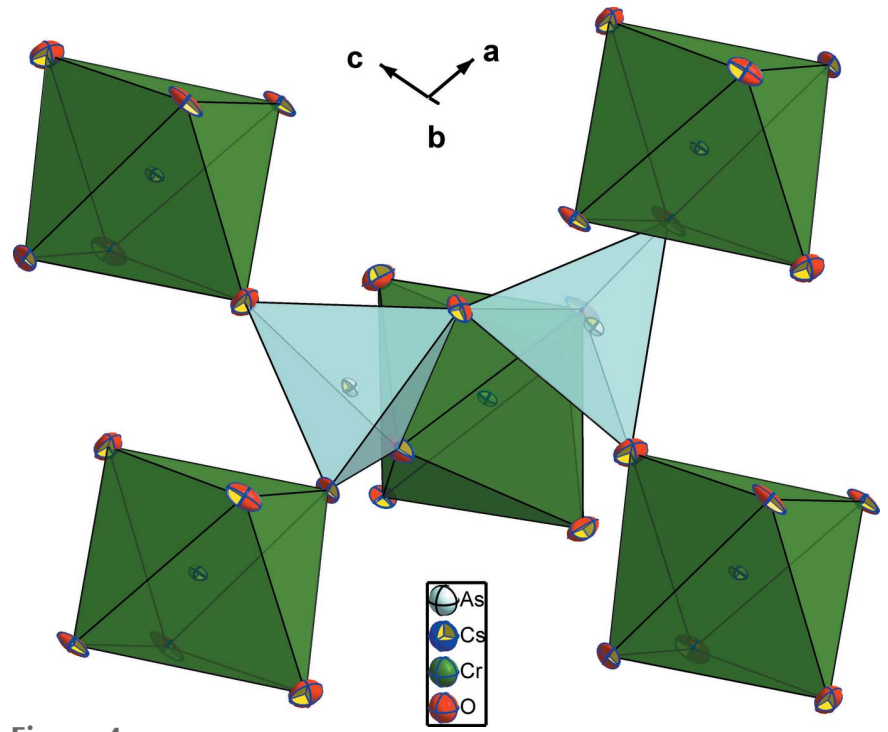

Figure 4

The environment of the diarsenate group in the structure of $\mathrm{CsCrAs}_{2} \mathrm{O}_{7}$. 


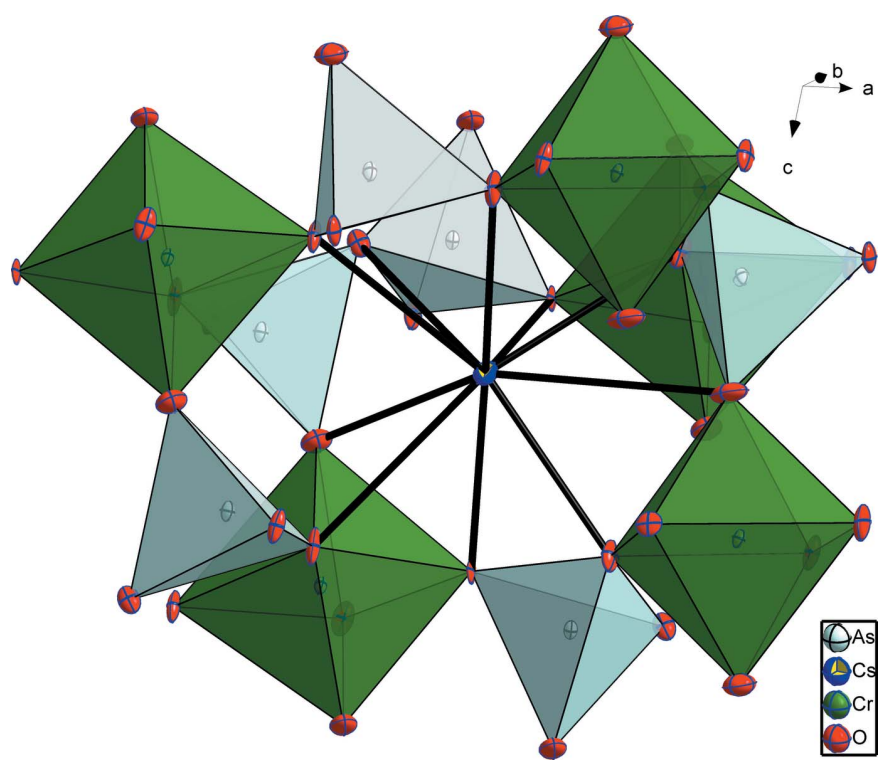

Figure 5

The surrounding of the ten-coordinated $\mathrm{Cs}^{+}$cation in the structure of $\mathrm{CsCrAs}_{2} \mathrm{O}_{7}$.

structure. In the title structure, belonging to the type I family of $A^{\mathrm{I}} M^{\mathrm{III}} X_{2} \mathrm{O}_{7}$ diarsenates, the diarsenate tetrahedra are in a nearly eclipsed conformation with a torsion angle O3-As1As2-O7 of $39.8(2)^{\circ}$, as shown in Fig. 6. The corresponding angle is $158.8(2)^{\circ}$ for $\mathrm{KAlAs}_{2} \mathrm{O}_{7}$ (Boughzala \& Jouini, 1995).

Using the bond-valence method (Brown, 2002), the calculated bond-valence-sum values (in valence units) of 5.08, 4.97, 3.01 and 1.35, respectively, for As1, As2, Cr and Cs are in good agreement with the expected oxidation states.

\section{Database survey}

The structure of $\mathrm{KAlP}_{2} \mathrm{O}_{7}(\mathrm{Ng} \&$ Calvo, 1973) was the first published of the $A^{\mathrm{I}} M^{\mathrm{III}} X_{2} \mathrm{O}_{7}$ family. Afterwards, based on different substitutions and combinations, a large number of

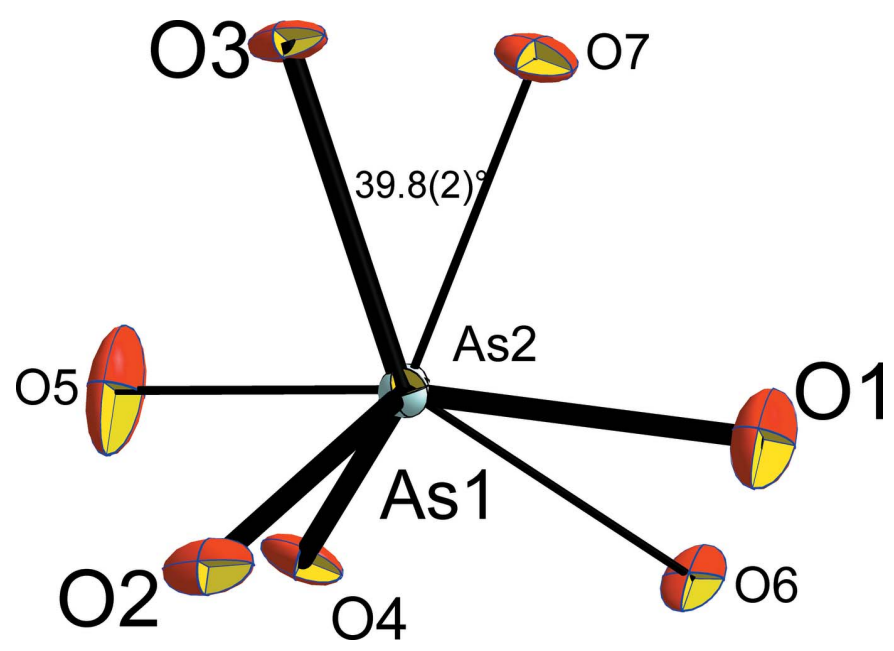

Figure 6

View parallel to the As1-As2 direction, emphasizing the nearly eclipsed conformation of the diarsenate anion.
Table 2

Experimental details.

\begin{tabular}{|c|c|}
\hline \multicolumn{2}{|l|}{ Crystal data } \\
\hline Chemical formula & $\mathrm{CsCrAs}_{2} \mathrm{O}_{7}$ \\
\hline$M_{\mathrm{r}}$ & 446.75 \\
\hline Crystal system, space group & Monoclinic, $P 2_{1} / c$ \\
\hline Temperature (K) & 293 \\
\hline$a, b, c(\AA)$ & $7.908(1), 10.0806(10), 8.6371(10)$ \\
\hline$\beta\left({ }^{\circ}\right)$ & $105.841(1)$ \\
\hline$V\left(\mathrm{~A}^{3}\right)$ & $662.38(13)$ \\
\hline$Z$ & 4 \\
\hline Radiation type & Мо $K \alpha$ \\
\hline$\mu\left(\mathrm{mm}^{-1}\right)$ & 17.05 \\
\hline Crystal size $(\mathrm{mm})$ & $0.20 \times 0.20 \times 0.10$ \\
\hline \multicolumn{2}{|l|}{ Data collection } \\
\hline Diffractometer & Enraf-Nonius CAD-4 \\
\hline Absorption correction & $\psi$ scan (North et al., 1968) \\
\hline$T_{\min }, T_{\max }$ & $0.132,0.281$ \\
\hline $\begin{array}{l}\text { No. of measured, independent and } \\
\text { observed }[I>2 \sigma(I)] \text { reflections }\end{array}$ & $1530,1433,1205$ \\
\hline$R_{\text {int }}$ & 0.051 \\
\hline$(\sin \theta / \lambda)_{\max }\left(\AA^{-1}\right)$ & 0.637 \\
\hline \multicolumn{2}{|l|}{ Refinement } \\
\hline$R\left[F^{2}>2 \sigma\left(F^{2}\right)\right], w R\left(F^{2}\right), S$ & $0.027,0.075,1.13$ \\
\hline No. of reflections & 1433 \\
\hline No. of parameters & 101 \\
\hline$\Delta \rho_{\max }, \Delta \rho_{\min }\left(\mathrm{e} \AA^{-3}\right)$ & $1.60,-1.23$ \\
\hline
\end{tabular}

Computer programs: CAD-4 EXPRESS (Enraf-Nonius, 1994), XCAD4 (Harms \& Wocadlo, 1995), SHELXS97 and SHELXL97 (Sheldrick, 2008), DIAMOND (Brandenburg, 2008) and publCIF (Westrip, 2010).

different phases were isolated and crystallographically characterized. Replacement of one of the cations can improve the structural and physical properties but also affects the coordination numbers, the degree of distortion of the coordination polyhedra and the conformation of the $X_{2} \mathrm{O}_{7}$ groups. Also, the crystal symmetry can be affected. The structures are triclinic, in space group $P \overline{1}$ with two formulas units, for the diarsenate compounds $A^{\mathrm{I}} \mathrm{AlAs}_{2} \mathrm{O}_{7}\left(A^{\mathrm{I}}=\mathrm{K}, \mathrm{Rb}, \mathrm{Tl}, \mathrm{Cs}\right)$ (Boughzala \& Jouini, 1992; Boughzala et al., 1993; Boughzala \& Jouini; 1995), whereas diphosphates are generally monoclinic. The isotypic $A^{\mathrm{I}} \mathrm{CrP}_{2} \mathrm{O}_{7}$ phases crystallize in space group $P 2_{1} / c$ for $A^{\mathrm{I}}=\mathrm{Na}$ (Bohaty et al., 1982), K (Gentil et al., 1997), $\mathrm{Rb}$ (Zhao \& Li, 2011) and Cs (Linde \& Gorbunova, 1982). The same counts for the $A^{\mathrm{I}} \mathrm{FeP}_{2} \mathrm{O}_{7}$ phases for $A^{\mathrm{I}}=\mathrm{Na}$ (GabelicaRobert et al., 1982) and K (Riou et al., 1988). However, the two Li-containing phases $\mathrm{Li} M \mathrm{P}_{2} \mathrm{O}_{7}$ show a symmetry reduction to space group $P 2_{1}(M=\mathrm{Cr}$, Ivashkevich et al., 2007; $M=\mathrm{Fe}$, Riou et al., 1990).

\section{Synthesis and crystallization}

The crystals of the title compound were obtained from heating a mixture of $\mathrm{Cs}_{2} \mathrm{CO}_{3}, \mathrm{Cr}_{2} \mathrm{O}_{3}$ and $\mathrm{NH}_{4} \mathrm{H}_{2} \mathrm{AsO}_{4}$, with a Cs:Cr:As molar ratio of 1:1:2. In order to eliminate volatile products, the sample was placed in a porcelain crucible and slowly heated under atmospheric conditions to $673 \mathrm{~K}$ and kept at that temperature for $24 \mathrm{~h}$. In a second step, the crucible was progressively heated at $1023 \mathrm{~K}$ for 4 days and then slowly cooled down at a rate of $5 \mathrm{~K} / 24 \mathrm{~h}$ to $923 \mathrm{~K}$ and finally 
quenched to room temperature. The product was washed with water and rinsed with an aqueous solution of $\mathrm{HCl}$. Two phases could be isolated. The major phase forms regular cube-shaped dark-green crystals of yet unknown composition. The second phase represents the title compound and was obtained in the form of pink crystals.

\section{Refinement}

Crystal data, data collection and structure refinement details are summarized in Table 2. The maximum and minimum electron density in the final difference Fourier map is located at $0.95 \AA, 0.87 \AA$, respectively, from the Cs atom.

\section{References}

Bohatý, L., Liebertz, J. \& Fröhlich, R. (1982). Z. Kristallogr. 161, 5359.

Boughzala, H., Driss, A. \& Jouini, T. (1993). Acta Cryst. C49, 425-427.

Boughzala, H. \& Jouini, T. (1992). C. R. Acad. Sci. Paris Ser. II, pp. 1419-1422.

Boughzala, H. \& Jouini, T. (1995). Acta Cryst. C51, 179-181.
Brandenburg, K. (2008). DIAMOND. Crystal Impact GbR, Bonn, Germany.

Brown, I. D. (2002). In he Chemical Bond in Inorganic Chemistry: The Bond Valence Model. Oxford University Press.

Enraf-Nonius (1994). CAD-4 EXPRESS. Enraf-Nonius, Delft, The Netherlands.

Gabelica-Robert, M., Goreaud, M., Labbe, Ph. \& Raveau, B. (1982). J. Solid State Chem. 45, 389-395.

Gentil, S., Andreica, D., Lujan, M., Rivera, J. P., Kubel, F. \& Schmid, H. (1997). Ferroelectrics, 204, 35-44.

Harms, K. \& Wocadlo, S. (1995). XCAD4. University of Marburg, Germany.

Ivashkevich, L. S., Selevich, K. A., Lesnikovich, A. I. \& Selevich, A. F. (2007). Acta Cryst. E63, i70-i72.

Linde, S. A. \& Gorbunova, Yu. E. (1982). Izv. Akad. Nauk SSSR Neorg. Mater. 18, 464-467.

Ng, H. N. \& Calvo, C. (1973). Can. J. Chem. 51, 2613-2620.

North, A. C. T., Phillips, D. C. \& Mathews, F. S. (1968). Acta Cryst. A24, 351-359.

Riou, D., Labbe, P. \& Goreaud, M. (1988). Eur. J. Solid. State Inorg. Chem. 25, 215-229.

Riou, D., Nguyen, N., Benloucif, R. \& Raveau, B. (1990). Mater. Res. Bull. 25, 1363-1369.

Sheldrick, G. M. (2008). Acta Cryst. A64, 112-122.

Westrip, S. P. (2010). J. Appl. Cryst. 43, 920-925.

Zhao, D. \& Li, F. (2011). Z. Kristallogr. 226, 443-444. 


\section{supporting information}

Acta Cryst. (2015). E71, 636-639 [doi:10.1107/S205698901500910X]

\section{Crystal structure of $\mathrm{CsCrAs}_{2} \mathrm{O}_{7}$, a new member of the diarsenate family}

\section{Mohamad Alem Bouhassine and Habib Boughzala}

\section{Computing details}

Data collection: CAD-4 EXPRESS (Enraf-Nonius, 1994); cell refinement: CAD-4 EXPRESS (Enraf-Nonius, 1994); data reduction: XCAD4 (Harms \& Wocadlo, 1995); program(s) used to solve structure: SHELXS97 (Sheldrick, 2008); program(s) used to refine structure: SHELXL97 (Sheldrick, 2008); molecular graphics: DIAMOND (Brandenburg, 2008); software used to prepare material for publication: publCIF (Westrip, 2010).

\section{Caesium chromium (III) diarsenate}

Crystal data

$\mathrm{CsCrAs}_{2} \mathrm{O}_{7}$

$M_{r}=446.75$

Monoclinic, $P 2_{1} / c$

Hall symbol: -P 2ybc

$a=7.908$ (1) $\AA$

$b=10.0806(10) \AA$

$c=8.6371(10) \AA$

$\beta=105.841(1)^{\circ}$

$V=662.38(13) \AA^{3}$

$Z=4$

\section{Data collection}

Enraf-Nonius CAD-4 diffractometer

Radiation source: fine-focus sealed tube Graphite monochromator $\omega / 2 \theta$ scans

Absorption correction: $\psi$ scan

(North et al., 1968)

$T_{\min }=0.132, T_{\max }=0.281$

1530 measured reflections

Refinement

Refinement on $F^{2}$

Least-squares matrix: full

$R\left[F^{2}>2 \sigma\left(F^{2}\right)\right]=0.027$

$w R\left(F^{2}\right)=0.075$

$S=1.13$

1433 reflections

101 parameters

0 restraints

Primary atom site location: structure-invariant direct methods
$F(000)=804$

$D_{\mathrm{x}}=4.480 \mathrm{Mg} \mathrm{m}^{-3}$

Mo $K \alpha$ radiation, $\lambda=0.71073 \AA$

Cell parameters from 25 reflections

$\theta=3.8-27^{\circ}$

$\mu=17.05 \mathrm{~mm}^{-1}$

$T=293 \mathrm{~K}$

Monoclinic, pink

$0.20 \times 0.20 \times 0.10 \mathrm{~mm}$

1433 independent reflections 1205 reflections with $I>2 \sigma(I)$

$R_{\text {int }}=0.051$

$\theta_{\max }=26.9^{\circ}, \theta_{\min }=2.7^{\circ}$

$h=-10 \rightarrow 9$

$k=0 \rightarrow 12$

$l=0 \rightarrow 11$

2 standard reflections every $120 \mathrm{~min}$ intensity decay: $1.1 \%$

Secondary atom site location: difference Fourier map

$w=1 /\left[\sigma^{2}\left(F_{0}^{2}\right)+(0.043 P)^{2}+1.1495 P\right]$

where $P=\left(F_{\mathrm{o}}{ }^{2}+2 F_{\mathrm{c}}{ }^{2}\right) / 3$

$(\Delta / \sigma)_{\max }<0.001$

$\Delta \rho_{\max }=1.60 \mathrm{e}^{-3}$

$\Delta \rho_{\min }=-1.23$ e $\AA^{-3}$

Extinction correction: SHELXL97 (Sheldrick, 2008), $\mathrm{Fc}^{*}=\mathrm{kFc}\left[1+0.001 \mathrm{xFc}^{2} \lambda^{3} / \sin (2 \theta)\right]^{-1 / 4}$

Extinction coefficient: 0.0018 (4) 


\section{Special details}

Geometry. All e.s.d.'s (except the e.s.d. in the dihedral angle between two 1.s. planes) are estimated using the full covariance matrix. The cell e.s.d.'s are taken into account individually in the estimation of e.s.d.'s in distances, angles and torsion angles; correlations between e.s.d.'s in cell parameters are only used when they are defined by crystal symmetry. An approximate (isotropic) treatment of cell e.s.d.'s is used for estimating e.s.d.'s involving 1.s. planes.

Refinement. Refinement of $F^{2}$ against ALL reflections. The weighted $R$-factor $w R$ and goodness of fit $S$ are based on $F^{2}$, conventional $R$-factors $R$ are based on $F$, with $F$ set to zero for negative $F^{2}$. The threshold expression of $F^{2}>\sigma\left(F^{2}\right)$ is used only for calculating $R$-factors (gt) etc. and is not relevant to the choice of reflections for refinement. $R$-factors based on $F^{2}$ are statistically about twice as large as those based on $F$, and $R$ - factors based on ALL data will be even larger.

Fractional atomic coordinates and isotropic or equivalent isotropic displacement parameters $\left(\AA^{2}\right)$

\begin{tabular}{lllll}
\hline & $x$ & $y$ & $z$ & $U_{\text {iso }} * U_{\text {eq }}$ \\
\hline As1 & $0.93130(7)$ & $0.13138(6)$ & $0.68578(6)$ & $0.00527(16)$ \\
As2 & $0.63567(7)$ & $0.08924(5)$ & $0.84021(6)$ & $0.00495(16)$ \\
Cs & $0.31839(5)$ & $0.19797(4)$ & $0.45751(4)$ & $0.01429(15)$ \\
Cr & $0.73911(11)$ & $0.39967(8)$ & $0.76602(10)$ & $0.0043(2)$ \\
O1 & $0.8001(6)$ & $0.1049(5)$ & $0.5039(5)$ & $0.0168(10)$ \\
O2 & $1.1355(5)$ & $0.0742(4)$ & $0.7202(5)$ & $0.0122(9)$ \\
O3 & $0.9413(5)$ & $0.2889(4)$ & $0.7514(5)$ & $0.0099(8)$ \\
O4 & $0.8363(5)$ & $0.0371(4)$ & $0.8122(5)$ & $0.0093(8)$ \\
O5 & $0.6538(6)$ & $0.0802(5)$ & $1.0338(5)$ & $0.0199(10)$ \\
O6 & $0.4833(5)$ & $-0.0082(4)$ & $0.7244(5)$ & $0.0085(8)$ \\
O7 & $0.5967(5)$ & $0.2403(4)$ & $0.7594(5)$ & $0.0109(8)$ \\
\hline
\end{tabular}

Atomic displacement parameters $\left(\AA^{2}\right)$

\begin{tabular}{lllllll}
\hline & $U^{11}$ & $U^{22}$ & $U^{33}$ & $U^{12}$ & $U^{13}$ & $U^{23}$ \\
\hline As1 & $0.0033(3)$ & $0.0057(3)$ & $0.0069(3)$ & $0.0003(2)$ & $0.0014(2)$ & $-0.0003(2)$ \\
As2 & $0.0034(3)$ & $0.0055(3)$ & $0.0059(3)$ & $-0.0009(2)$ & $0.0013(2)$ & $-0.0002(2)$ \\
Cs & $0.0106(2)$ & $0.0165(2)$ & $0.0136(2)$ & $-0.00201(14)$ & $-0.00033(14)$ & $0.00299(14)$ \\
Cr & $0.0026(4)$ & $0.0045(4)$ & $0.0055(4)$ & $0.0004(3)$ & $0.0005(3)$ & $-0.0002(3)$ \\
O1 & $0.017(2)$ & $0.022(2)$ & $0.010(2)$ & $-0.0029(19)$ & $0.0010(17)$ & $-0.0022(18)$ \\
O2 & $0.0044(19)$ & $0.007(2)$ & $0.025(2)$ & $-0.0020(16)$ & $0.0035(16)$ & $-0.0024(17)$ \\
O3 & $0.0040(18)$ & $0.0045(19)$ & $0.020(2)$ & $0.0006(15)$ & $0.0012(16)$ & $-0.0032(16)$ \\
O4 & $0.0077(19)$ & $0.0067(19)$ & $0.015(2)$ & $0.0038(15)$ & $0.0049(15)$ & $0.0035(16)$ \\
O5 & $0.016(2)$ & $0.035(3)$ & $0.007(2)$ & $-0.009(2)$ & $0.0016(17)$ & $-0.0009(18)$ \\
O6 & $0.0065(19)$ & $0.008(2)$ & $0.011(2)$ & $-0.0061(16)$ & $0.0024(15)$ & $-0.0007(16)$ \\
O7 & $0.0074(19)$ & $0.0047(19)$ & $0.020(2)$ & $-0.0012(16)$ & $0.0018(15)$ & $0.0030(17)$ \\
\hline
\end{tabular}

Geometric parameters $\left(\stackrel{A}{\circ}{ }^{\circ}\right)$

\begin{tabular}{llll}
\hline $\mathrm{Cs}-\mathrm{O} 7$ & $2.948(4)$ & $\mathrm{Cr}-\mathrm{O} 1^{\mathrm{v}}$ & $1.978(4)$ \\
$\mathrm{Cs}-\mathrm{O} 3^{\mathrm{i}}$ & $3.030(4)$ & $\mathrm{Cr}-\mathrm{O} 3$ & $1.982(4)$ \\
$\mathrm{Cs}-\mathrm{O} 6$ & $3.113(4)$ & $\mathrm{Cr}-\mathrm{O} 2^{\mathrm{vi}}$ & $2.007(4)$ \\
$\mathrm{Cs}-\mathrm{O}^{\mathrm{ii}}$ & $3.152(4)$ & $\mathrm{Cr}-\mathrm{O} 6^{\mathrm{vi}}$ & $2.010(4)$ \\
$\mathrm{Cs}-\mathrm{O}^{\mathrm{i}}$ & $3.155(4)$ & $\mathrm{As} 1-\mathrm{O} 1$ & $1.651(4)$ \\
$\mathrm{Cs}-\mathrm{O} 7^{\mathrm{iii}}$ & $3.196(4)$ & $\mathrm{As} 1-\mathrm{O} 2$ & $1.664(4)$ \\
$\mathrm{Cs}-\mathrm{O}^{\mathrm{ii}}$ & $3.237(5)$ & $\mathrm{As} 1-\mathrm{O} 3$ & $1.681(4)$
\end{tabular}




\begin{tabular}{|c|c|c|c|}
\hline $\mathrm{Cs}-\mathrm{O} 2^{\mathrm{iv}}$ & $3.253(4)$ & As $1-\mathrm{O} 4$ & $1.763(4)$ \\
\hline $\mathrm{Cs}-\mathrm{O} 4^{\mathrm{ii}}$ & $3.314(4)$ & As $2-\mathrm{O} 5$ & $1.641(4)$ \\
\hline $\mathrm{Cs}-\mathrm{O} 5^{\mathrm{iii}}$ & $3.393(5)$ & As2-O6 & $1.661(4)$ \\
\hline $\mathrm{Cr}-\mathrm{O} 5^{\mathrm{iii}}$ & $1.944(4)$ & As $2-07$ & $1.669(4)$ \\
\hline $\mathrm{Cr}-\mathrm{O} 7$ & $1.954(4)$ & As $2-\mathrm{O} 4$ & $1.750(4)$ \\
\hline $\mathrm{O} 1-\mathrm{As} 1-\mathrm{O} 2$ & $116.2(2)$ & $\mathrm{O} 6^{\mathrm{ii}}-\mathrm{Cs}-\mathrm{O} 5^{\mathrm{iii}}$ & $91.60(11)$ \\
\hline $\mathrm{O} 1-\mathrm{As} 1-\mathrm{O} 3$ & $115.7(2)$ & $\mathrm{O} 2^{\mathrm{i}}-\mathrm{Cs}-\mathrm{O} 5^{\mathrm{iii}}$ & 80.89 (11) \\
\hline $\mathrm{O} 2-\mathrm{As} 1-\mathrm{O} 3$ & $108.3(2)$ & $\mathrm{O} 7^{\mathrm{iii}}-\mathrm{Cs}-\mathrm{O}^{\mathrm{iii}}$ & $50.19(10)$ \\
\hline $\mathrm{O} 1-\mathrm{As} 1-\mathrm{O} 4$ & $103.8(2)$ & $\mathrm{O} 1^{\mathrm{ii}}-\mathrm{Cs}-\mathrm{O} 5^{\mathrm{iii}}$ & $146.54(11)$ \\
\hline $\mathrm{O} 2-\mathrm{As} 1-\mathrm{O} 4$ & $105.1(2)$ & $\mathrm{O} 2^{\mathrm{iv}}-\mathrm{Cs}-\mathrm{O} 5^{\mathrm{iii}}$ & $126.15(10)$ \\
\hline $\mathrm{O} 3-\mathrm{As} 1-\mathrm{O} 4$ & $106.8(2)$ & $\mathrm{O} 4^{\mathrm{ii}}-\mathrm{Cs}-\mathrm{O} 5^{\mathrm{iii}}$ & $136.61(10)$ \\
\hline $\mathrm{O} 5-\mathrm{As} 2-\mathrm{O} 6$ & $115.3(2)$ & $\mathrm{O} 5{ }^{\mathrm{iii}}-\mathrm{Cr}-\mathrm{O} 7$ & $91.2(2)$ \\
\hline $\mathrm{O} 5-\mathrm{As} 2-\mathrm{O} 7$ & $115.6(2)$ & $\mathrm{O} 5^{\mathrm{iii}}-\mathrm{Cr}-\mathrm{O} 1^{\mathrm{v}}$ & 172.37 (19) \\
\hline $\mathrm{O} 6-\mathrm{As} 2-\mathrm{O} 7$ & $105.5(2)$ & $\mathrm{O} 7-\mathrm{Cr}-\mathrm{O}^{\mathrm{v}}$ & $89.22(18)$ \\
\hline $\mathrm{O} 5-\mathrm{As} 2-\mathrm{O} 4$ & $107.1(2)$ & $\mathrm{O} 5^{\mathrm{iii}}-\mathrm{Cr}-\mathrm{O} 3$ & 92.97 (19) \\
\hline $\mathrm{O} 6-\mathrm{As} 2-\mathrm{O} 4$ & 105.98 (19) & $\mathrm{O} 7-\mathrm{Cr}-\mathrm{O} 3$ & $90.21(17)$ \\
\hline $\mathrm{O} 7-\mathrm{As} 2-\mathrm{O} 4$ & 106.69 (19) & $\mathrm{O} 1^{\mathrm{v}}-\mathrm{Cr}-\mathrm{O} 3$ & 94.65 (19) \\
\hline $\mathrm{O} 7-\mathrm{Cs}-\mathrm{O}^{\mathrm{i}}$ & $152.96(12)$ & $\mathrm{O} 5^{\mathrm{iii}}-\mathrm{Cr}-\mathrm{O} 2^{\mathrm{vi}}$ & $89.8(2)$ \\
\hline $\mathrm{O} 7-\mathrm{Cs}-\mathrm{O} 6$ & $51.76(11)$ & $\mathrm{O} 7-\mathrm{Cr}-\mathrm{O} 2^{\mathrm{vi}}$ & $173.72(17)$ \\
\hline $\mathrm{O} 3^{\mathrm{i}}-\mathrm{Cs}-\mathrm{O} 6$ & $127.61(10)$ & $\mathrm{O} 1^{\mathrm{v}}-\mathrm{Cr}-\mathrm{O} 2^{\mathrm{vi}}$ & 88.99 (19) \\
\hline $\mathrm{O} 7-\mathrm{Cs}-\mathrm{O}^{\mathrm{ii}}$ & $100.13(11)$ & $\mathrm{O} 3-\mathrm{Cr}-\mathrm{O} 2^{\mathrm{vi}}$ & 95.94 (17) \\
\hline $\mathrm{O} 3^{\mathrm{i}}-\mathrm{Cs}-\mathrm{O}^{\mathrm{ii}}$ & $106.10(11)$ & $\mathrm{O} 5^{\mathrm{iii}}-\mathrm{Cr}-\mathrm{O}^{\mathrm{vii}}$ & $86.15(18)$ \\
\hline $\mathrm{O} 6-\mathrm{Cs}-\mathrm{O}^{\mathrm{ii}}$ & $78.42(11)$ & $\mathrm{O} 7-\mathrm{Cr}-\mathrm{O}^{\mathrm{vii}}$ & $82.96(18)$ \\
\hline $\mathrm{O} 7-\mathrm{Cs}-\mathrm{O} 2^{\mathrm{i}}$ & $124.64(11)$ & $\mathrm{O} 1^{\mathrm{v}}-\mathrm{Cr}-\mathrm{O}^{\mathrm{vii}}$ & $86.33(18)$ \\
\hline $\mathrm{O} 3^{\mathrm{i}}-\mathrm{Cs}-\mathrm{O} 2^{\mathrm{i}}$ & $51.93(11)$ & $\mathrm{O} 3-\mathrm{Cr}-\mathrm{O}^{\mathrm{vii}}$ & $173.08(17)$ \\
\hline $\mathrm{O} 6-\mathrm{Cs}-\mathrm{O}^{2}$ & $172.88(11)$ & $\mathrm{O} 2^{\mathrm{vi}}-\mathrm{Cr}-\mathrm{O} 6^{\mathrm{vii}}$ & $90.92(17)$ \\
\hline $\mathrm{O} 6^{\mathrm{ii}}-\mathrm{Cs}-\mathrm{O} 2^{\mathrm{i}}$ & $108.67(11)$ & $\mathrm{As} 1-\mathrm{O} 1-\mathrm{Cr}^{\mathrm{iii}}$ & $154.6(3)$ \\
\hline $\mathrm{O} 7-\mathrm{Cs}-\mathrm{O}^{\mathrm{iii}}$ & $89.34(11)$ & $\mathrm{As} 1-\mathrm{O} 1-\mathrm{Cs}^{\mathrm{ii}}$ & 100.29 (19) \\
\hline $\mathrm{O} 3^{\mathrm{i}}-\mathrm{Cs}-\mathrm{O}^{\mathrm{iii}}$ & $112.83(12)$ & $\mathrm{Cr}^{\mathrm{iii}}-\mathrm{O} 1-\mathrm{Cs}^{\mathrm{ii}}$ & $95.14(16)$ \\
\hline $\mathrm{O} 6-\mathrm{Cs}-\mathrm{O}^{\mathrm{iii}}$ & $108.42(11)$ & $\mathrm{As} 1-\mathrm{O} 2-\mathrm{Cr}^{\mathrm{viii}}$ & $139.0(2)$ \\
\hline $\mathrm{O}^{\mathrm{ii}}-\mathrm{Cs}-\mathrm{O} 7^{\mathrm{iii}}$ & $48.86(11)$ & $\mathrm{As} 1-\mathrm{O} 2-\mathrm{Cs}^{\mathrm{ix}}$ & $96.55(17)$ \\
\hline $\mathrm{O} 2^{\mathrm{i}}-\mathrm{Cs}-\mathrm{O}^{\mathrm{iii}}$ & $76.75(11)$ & $\mathrm{Cr}^{\mathrm{viii}}-\mathrm{O} 2-\mathrm{Cs}^{\mathrm{ix}}$ & $117.92(17)$ \\
\hline $\mathrm{O} 7-\mathrm{Cs}-\mathrm{O} 1^{\mathrm{ii}}$ & $102.27(11)$ & $\mathrm{As} 1-\mathrm{O} 2-\mathrm{Cs}^{\mathrm{x}}$ & $109.71(19)$ \\
\hline $\mathrm{O} 3^{\mathrm{i}}-\mathrm{Cs}-\mathrm{O} 1^{\mathrm{ii}}$ & $80.54(11)$ & $\mathrm{Cr}^{\mathrm{viii}}-\mathrm{O} 2-\mathrm{Cs}^{\mathrm{x}}$ & 94.09 (15) \\
\hline $\mathrm{O} 6-\mathrm{Cs}-\mathrm{O}^{\mathrm{ii}}$ & $50.85(11)$ & $\mathrm{Cs}^{\mathrm{ix}}-\mathrm{O} 2-\mathrm{Cs}^{\mathrm{x}}$ & $87.81(10)$ \\
\hline $\mathrm{O} 6^{\mathrm{ii}}-\mathrm{Cs}-\mathrm{O} 1^{\mathrm{ii}}$ & $71.11(11)$ & $\mathrm{As} 1-\mathrm{O} 3-\mathrm{Cr}$ & $126.1(2)$ \\
\hline $\mathrm{O} 2^{\mathrm{i}}-\mathrm{Cs}-\mathrm{O} 1^{\mathrm{ii}}$ & $131.26(11)$ & $\mathrm{As} 1-\mathrm{O} 3-\mathrm{Cs}^{\mathrm{ix}}$ & $100.80(17)$ \\
\hline $\mathrm{O} 7^{\mathrm{iii}}-\mathrm{Cs}-\mathrm{O} 1^{\mathrm{ii}}$ & $119.98(11)$ & $\mathrm{Cr}-\mathrm{O} 3-\mathrm{Cs}^{\mathrm{ix}}$ & $128.28(18)$ \\
\hline $\mathrm{O} 7-\mathrm{Cs}-\mathrm{O} 2^{\mathrm{iv}}$ & $78.78(11)$ & As2-O4-As1 & $118.7(2)$ \\
\hline $\mathrm{O} 3^{\mathrm{i}}-\mathrm{Cs}-\mathrm{O} 2^{\mathrm{iv}}$ & $82.70(11)$ & $\mathrm{As} 2-\mathrm{O} 4-\mathrm{Cs}^{\mathrm{ii}}$ & $97.91(16)$ \\
\hline $\mathrm{O} 6-\mathrm{Cs}-\mathrm{O} 2^{\mathrm{iv}}$ & $53.39(11)$ & $\mathrm{As} 1-\mathrm{O} 4-\mathrm{Cs}^{\mathrm{ii}}$ & $95.06(16)$ \\
\hline $\mathrm{O} 6^{\mathrm{ii}}-\mathrm{Cs}-\mathrm{O} 2^{\mathrm{iv}}$ & $119.42(10)$ & As $2-\mathrm{O} 5-\mathrm{Cr}^{\mathrm{v}}$ & $162.6(3)$ \\
\hline $\mathrm{O} 2^{\mathrm{i}}-\mathrm{Cs}-\mathrm{O} 2^{\mathrm{iv}}$ & $121.35(13)$ & As $2-\mathrm{O} 5-\mathrm{Cs}^{\mathrm{v}}$ & 85.37 (19) \\
\hline $\mathrm{O} 7^{\mathrm{iii}}-\mathrm{Cs}-\mathrm{O} 2^{\mathrm{iv}}$ & $161.82(10)$ & $\mathrm{Cr}-\mathrm{O} 5-\mathrm{Cs}^{\mathrm{v}}$ & $99.46(18)$ \\
\hline 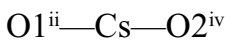 & $50.97(11)$ & As $2-\mathrm{O} 6-\mathrm{Cr}^{\mathrm{xi}}$ & $138.4(2)$ \\
\hline $\mathrm{O} 7-\mathrm{Cs}-\mathrm{O} 4^{\mathrm{ii}}$ & $140.21(11)$ & As2-O6- $-\mathrm{Cs}$ & $98.02(17)$ \\
\hline $\mathrm{O} 3^{\mathrm{i}}-\mathrm{Cs}-\mathrm{O} 4^{\mathrm{ii}}$ & $60.20(10)$ & $\mathrm{Cr}^{\mathrm{xi}}-\mathrm{O} 6-\mathrm{Cs}$ & $98.31(15)$ \\
\hline
\end{tabular}




$\begin{array}{llll}\mathrm{O} 6-\mathrm{Cs}-\mathrm{O} 4^{\mathrm{ii}} & 92.45(11) & \mathrm{As} 2-\mathrm{O} 6-\mathrm{Cs}^{\mathrm{ii}} & 106.32(18) \\ \mathrm{O} 6^{\mathrm{ii}}-\mathrm{Cs}-\mathrm{O} 4^{\mathrm{ii}} & 49.76(10) & \mathrm{Cr}-\mathrm{O} 6-\mathrm{Cs}^{\mathrm{ii}} & 107.45(16) \\ \mathrm{O} 2^{\mathrm{i}}-\mathrm{Cs}-\mathrm{O}^{\mathrm{ii}} & 92.76(11) & \mathrm{Cs}-\mathrm{O}-\mathrm{Cs}^{\mathrm{ii}} & 101.58(11) \\ \mathrm{O} 7^{\mathrm{iii}}-\mathrm{Cs}-\mathrm{O} 4^{\mathrm{ii}} & 86.49(10) & \mathrm{As} 2-\mathrm{O} 7-\mathrm{Cr} & 134.3(2) \\ \mathrm{O} 1^{\mathrm{ii}}-\mathrm{Cs}-\mathrm{O} 4^{\mathrm{ii}} & 48.42(10) & \mathrm{As} 2-\mathrm{O} 7-\mathrm{Cs} & 115.48(17) \\ \mathrm{O} 2^{\mathrm{iv}}-\mathrm{Cs}-\mathrm{O} 4^{\mathrm{ii}} & 93.83(10) & \mathrm{Cr}-\mathrm{O} 7-\mathrm{Cs} & 91.67(16) \\ \mathrm{O} 7-\mathrm{Cs}-\mathrm{O} 5^{\mathrm{iii}} & 51.51(11) & \mathrm{As} 2-\mathrm{O} 7-\mathrm{Cs}^{\mathrm{v}} & 107.45(17) \\ \mathrm{O} 3^{\mathrm{i}}-\mathrm{Cs}-\mathrm{O} 5^{\mathrm{iii}} & 132.61(11) & \mathrm{Cr}-\mathrm{O} 7-\mathrm{Cs}^{\mathrm{v}} & 92.57(11) \\ \mathrm{O} 6-\mathrm{Cs}-\mathrm{O} 5^{\mathrm{iii}} & 98.57(11) & \mathrm{Cs}-\mathrm{O} 7-\mathrm{Cs}^{\mathrm{v}} & \end{array}$

Symmetry codes: (i) $x-1,-y+1 / 2, z-1 / 2$; (ii) $-x+1,-y,-z+1$; (iii) $x,-y+1 / 2, z-1 / 2$; (iv) $x-1, y, z$; (v) $x,-y+1 / 2, z+1 / 2$; (vi) $-x+2, y+1 / 2,-z+3 / 2$; (vii) $-x+1, y+1 / 2,-z+3 / 2$; (viii) $-x+2, y-1 / 2,-z+3 / 2$; (ix) $x+1,-y+1 / 2, z+1 / 2$; (x) $x+1, y, z$; (xi) $-x+1, y-1 / 2,-z+3 / 2$. 